\title{
Amicitia y relaciones clientelares durante el Bellum Hispaniense
}

\section{Amicitia and clientelar relations during the Bellum Hispaniense}

\author{
Miguel Ángel Novillo LóPeZ*
}

\begin{abstract}
RESUMEN
ABSTRACT

Las relaciones de amicitia y clientela pompeyanas y cesarianas durante el

This paper aims to show how friendship relations and the Pompeian and Caesarian clientelae shaped the political Bellum Hispaniense confirman cómo son éstas las que definen en última instancia los modelos de gestión política y territorial en la península Ibérica. and territorial organization in the Iberian Peninsula during the Bellum Hispaniense.

PALABRAS CLAVE:

KEYWORDS:

Bellum Hispaniense, pompeyanos, cesarianos, amicitia, clientela, clemencia, Bellum Hispaniense, Pompeians, deserción. Caesarians, amicitia, clientela, clemency, desertion.
\end{abstract}

\section{INTRODUCCIÓN}

La amicitia y el fenómeno clientelar, interpretados como vínculos interpersonales de apoyo y colaboración, así como relaciones de dependencia personal entre individuos de la misma o distinta condición social que buscaban un bien recíproco, fueron fenómenos muy extendidos durante el siglo I a.C. presidiendo el funcionamiento de la vida pública y política de Roma. Tales fenómenos se dieron con Cn. Pompeyo Magno y C. Julio César, quienes aprovecharon el ejercicio de

* Departamento de Historia Antigua, Universidad Complutense de Madrid (C/ Profesor Aranguren, s/n. 28040 Madrid). E-mail: ma_novillo_17@ hotmail.com.

Artículo basado en la comunicación leída el 20 de Mayo de 2008, en la VII edición del Encuentro de Jóvenes Investigadores de Historia Antigua de la Universidad Complutense de Madrid. 
sus magistraturas y sus amistades para poder forjar auténticas redes clientelares con objeto de poder confirmar su poder e influencia, haciendo de éstas un sólido soporte o apoyo de su poder personal. No obstante, entre las gestiones pompeyanas y cesarianas se observan diferencias tanto en los procedimientos como en los objetivos. Por ello, es necesario ver qué intereses guardaban cada una de las dos partes así como qué derechos y obligaciones existían. Es necesario, además, definir los contextos sociales, económicos y culturales en los que se manifestaron.

A priori, en las gestiones pompeyanas se observa la carencia de un modelo de transformación de la administración local a gran escala. En cierto sentido, seguía anquilosado en los modelos tradicionales de gobierno limitándose a mantener relaciones de amicitia y de clientela con las oligarquías locales. Su interés se fundamentaba en poder mantener controladas a su servicio a estas oligarquías dotándolas de cierto grado de autonomía. Ahora bien, es necesario determinar el porqué de sus intenciones, ya que hay que confirmar si sus planes respondían al diseño de un nuevo modelo administrativo o a la búsqueda de una sumisión política y militar. Por medio de las relaciones de amicitia y de clientela, César buscaba una reorganización e integración del territorio hispano aplicando el modelo de la civitas romana. Buscaba la disolución de los conjuntos étnicos y agrupaciones de colectivos indígenas basados en el caciquismo aplicando nuevos modelos de reclutamiento e integración político-administrativa. En ambos casos se llega a la conclusión de que la clientela provincial derivó de la influencia personal que tenían los distintos gobernadores provinciales de turno en un área concreta.

En el análisis que pretendemos llevar a cabo buscamos dar respuesta a una serie de cuestiones: ¿qué similitudes y diferencias existen entre las amistades y las clientelas pompeyanas y cesarianas? ¿Es la amistad una forma de cohesión social entre las oligarquías o colectivos dirigentes con el mandatario de turno? ¿Qué es lo que mueve a forjar estas redes clientelares? ¿Cuáles son los medios en los que se apoyan los generales romanos para mantener un sistema clientelar lo suficientemente sólido como para mantenerse en el poder? ¿Podemos concebir la clientela o el patronazgo como la fórmula por medio de la cual las grandes familias permanecían siempre en el poder? ¿La concesión de privilegios en este momento responde a un programa oficial definido o a la acción política de personajes concretos? ¿En qué casos estas relaciones se circunscriben a las oligarquías locales y en cuáles a toda la población?.

\section{ACLARACIONES TERMINOLÓGICAS}

La amicitia significó para la sociedad romana amistad, amor, benevolencia, familiaridad, cariño, afinidad o confianza recíproca, siendo un término utilizado para designar la alianza y la concordia entre dos o más miembros o colectivos, es decir, la amistad política. La amicitia suponía una relación social de apoyo, colaboración y dependencia entre iguales aunque no de forma obligatoria, que en ocasiones po- 
día ser hereditaria ${ }^{1}$. En este sentido, los pactos de amicitia se habían convertido en una práctica común entre las ciudades libres de las provincias y Roma.

Quizás el ejemplo de amicitia más célebre del periodo tardorrepublicano sea el sellado por Pompeyo, Craso y César, comúnmente denominado como I Triunvirato². Tanto Pompeyo como César estaban ansiosos de hacerse con el poder en Roma. Pronto comprendieron que la única vía posible para conseguirlo era por medio de la alianza política creando vínculos de amistad. No se trataba sino de una relación de amicitia en la que Pompeyo aportaba el potencial de sus ejércitos, Craso su potencial económico y su influencia en determinados círculos del Senado, y César el apoyo de los populares y los ulteriores poderes si accedía al consulado ${ }^{3}$. Si César accedía a éste, debía conseguir del Senado la ratificación de las medidas adoptadas por Pompeyo en su reorganización en Oriente, y conseguir para Craso un mayor enriquecimiento y apoyo de los publicani. Después de varios sobornos, los planes de los triunviros se vieron cumplidos cuando en 59 a.C. César fue elegido cónsul junto con M. Calpurnio Bíbulo. Las relaciones entre los triunviros se vieron reforzadas por medio de alianzas matrimoniales cuando César ofreció la mano de su hija Julia a Pompeyo. Sin embargo, el propio devenir de la República traería consigo la ruptura entre las partes contratantes ${ }^{4}$.

Para Hellegouarc'h ${ }^{5}$, los términos de familiaritas, necessitudo y consuetudo son los términos que más frecuentemente aparecen en las relaciones de amicitia. Marcial ${ }^{6}$ parece especialmente proclive a utilizar sodalitas para referirse a los lazos asimilables a la amicitia. Otra particularidad es el empleo del término rex para designar al magnus amicus que asume el rol patronal. Por su parte, Plinio ${ }^{7}$ alude en repetidas ocasiones a los officia amicitiae. Por otro lado, la clientela comprendía cualquier tipo de protección o gracia que una persona poderosa daba a otra, de igual o de menor condición social, a cambio de fidelidad. El vínculo ejercido por la clientela supuso la existencia de una parte dominante y una parte dependiente, destinada esta última a obedecer en paz y en guerra con la obligación de la recíproca buena fe.

Debemos reconocer toda distinción entre amicus y cliens. Aunque en principio ambas palabras se aplican en situaciones muy diferentes, en el uso se volvieron intercambiables a merced de las necesidades y las circunstancias del momento. No hemos de confundir tampoco la relación clientelar con la institución del hospitium,

1 Para J. M. Serrano (1989: 177-78), la amicitia es una relación tan funcional y respetada que es capaz de mediar en las relaciones entre patrono-cliente amparándose en la ley.

2 Llamado así erróneamente por tratarse de un pacto privado y secreto entre las tres partes que escapaba de los límites de toda legalidad posible.

3 Rawson (1978: 148-49).

4 Caes. Ciu. 1.4: «El mismo Pompeyo, incitado por los enemigos de César y porque no estaba dispuesto a que nadie se igualara con él en dignidad, se había apartado completamente de su amistad, había vuelto a ser amigo de los enemigos comunes, a la mayoría de los cuales el propio Pompeyo, en la época aquella de parentesco, había enemistado con César». César también sellaría pactos de amistad con personajes de suma relevancia como Publio Cornelio Léntulo Espínter o Labieno.

5 J. Hellegouarc'h (1972: 68-71).

6 Marcial Epigr. 1.15, 1.54, 3.7, 5.22.

7 Plin. Ep. 1.13. 
ni esta última con la amicitia por presentar unas particularidades más precisas, pues mientras que el hospitium es un pacto sinalagmático que promete a quienes lo contraen protección recíproca, la relación clientelar trae consigo una relación de subordinación mediante la cual, y en virtud de una deditio, applicatio, o manumissio, la parte más débil se confía a otra más fuerte mediante una relación de beneficia-officia ${ }^{8}$.

Para L. R. Taylor, el fenómeno de la amicitia viene caracterizado por la cualidad de festividad-amistad. R. Syme sostuvo que la amicitia era un arma fundamentalmente política y no un sentimiento basado en la congenialidad de las partes implicadas. Para P. A. Brunt, si un hombre llamaba a otro amicus, esto traía consigo una fuerte connotación de gran contenido político y social durante época tardorrepublicana ${ }^{9}$. En lo que respecta a los vínculos de amicitia forjados tanto por Pompeyo como por César durante el transcurso del conflicto, lo que diferenció a uno y otro fue que, a diferencia de Pompeyo, quien guardó pactos de amicitia a nivel individual, César logró la amicitia con grandes colectivos y comunidades ${ }^{10}$.

\section{CLIENTELA Y AMICITIA}

Hispania fue siempre concebida como una fuente inagotable de recursos naturales y de tropas auxiliares. Fue F. de Coulanges quien puso de relieve la importancia de la clientela y de la amicitia romana, ya que a través de la clientela y las amistades las grandes familias de Roma mantenían su poder ${ }^{11}$. En época tardorrepublicana la concesión de la ciudadanía romana no obedeció a un programa oficial, sino a la acción política puntual de determinados generales al amparo de la legislación ordinaria y de sus poderes extraordinarios. En este sentido, el Senado se mostró mucho más avaro en la concesión de la ciudadanía romana en las provincias que en Italia, sobre todo para evitar que los diferentes patrones pudieran otorgar el derecho de civitas a sus clientes.

Hasta el momento no contamos con noticias sobre la existencia de vínculos interpersonales en la Hispania prerromana análogos a los de la clientela romana, pues los pactos de hospitalidad no son equiparables con esta institución ${ }^{12}$. Tanto Pompeyo como César utilizaron en su propio beneficio las redes clientelares y las de amicitia con objeto de hacer más fuerte su posición política y militar en Hispania. En lo que respecta a las clientelas privadas, podemos distinguir tres momentos en su transformación: 1. Los clientes de los patricios en el comienzo de la Historia de Roma. 2. Las guerras civiles trajeron consigo lazos de todo tipo, procediendo al abandono del compromiso de carácter jurídico para acordar un compromiso de carácter moral, ya que lo que realmente imperaba era seguir manteniendo el necesario acuerdo social. En este momento incluso los libertos podían ser clientes y, a

\footnotetext{
8 Nicols (1980: 551).

9 Brunt (1965: 199).

${ }_{10}$ Ciu. 1.48, 1.52, 1.60, 3.9, 3.56, 3.59.

11 De Coulanges (1890).

12 Mangas (1978: 217).
} 
su vez, podían ser clientes de varios patronos. A cambio de demostrar su fides, el cliente recibía de su patrono protección, favores y congratulaciones varias. No obstante, el afianzamiento de las medidas valedoras a la plebe a cargo del Estado contribuyó decisivamente a la disminución numérica de la clientela privada y al relajamiento de los antiguos vínculos ${ }^{13}$. 3. La naturaleza de institución regresiva de la clientela privada se fijó primordialmente en época imperial.

La concesión del estatuto privilegiado a las comunidades provinciales obligaba a las oligarquías locales a tener que sufragar gastos de todo tipo. El carácter regresivo de la clientela privada en los primeros años del Imperio y el debilitamiento de los compromisos que implicaba abrió paso al desarrollo de las relaciones interpersonales de amicitia, observándose que el fenómeno de la clientela privada en época imperial fue mayor que en época republicana ${ }^{14}$.

\section{RELACIONES POMPEYANAS DURANTE EL CONFLICTO}

Los antecedentes de la clientela pompeyana en Hispania hay que buscarlos en la clientela que su progenitor, Cn. Pompeyo Estrabón, logró crear con los miembros de la turma Salluitana a los que concedió la ciudadanía en 89 a.C. por los servicios y la fidelidad mostrada durante el transcurso de las Guerras Sociales. En realidad habría que considerar a Q. Pompeyo (cos. 141 a.C.) como el pionero de la clientela pompeyana al fijar los cimientos sobre los que posteriormente Pompeyo forjaría una auténtica red clientelar de gran calado, al ser $Q$. Pompeyo quien comienza a entablar contacto con los jefes indígenas de la región del valle del Ebro dando a conocer el nombre de su gens en esta zona. La concesión de la ciudadanía romana a los miembros de la turma Salluitana respondió a factores de índole social, administrativa y militar $^{15}$.

Pompeyo heredó las clientelas y patronazgos que su padre tenía en el Piceno y en la Galia Cisalpina ${ }^{16}$. No resultaría osado pensar que a su vez heredase las clientelas de Craso al contar en este momento con el poder y los apoyos senatoriales suficientes como para poder acapararlas. Si interpretamos el Piceno como base de las operaciones en su carrera clientelar, podemos distinguir entonces tres fases o escenarios de operaciones ${ }^{17}:$ 1. Galia Cisalpina, Sicilia y África. 2. Galia Transalpina e Hispania. 3. Oriente. A raíz del conflicto sertoriano, Pompeyo comenzó a crear una sólida y amplia red clientelar y de amistad en la Hispania Citerior. Los mecanismos que empleó fueron los mismos que aplicó en Sicilia y en África años atrás, como la concesión de bienes materiales para ganarse así el apoyo y la voluntad de las oligarquías indígenas de las zonas menos romanizadas o la concesión de la ciudadanía a título individual en las zonas más romanizadas.

13 Yavetz (1958).

14 Mangas (1978: 219).

15 Amela (2000: 87).

16 Dio Cass. 33.107; Plut. Pomp. 6.1.

17 Badian (1958: 278). 
Como gobernador de la Hispania Citerior entre 77-71 a.C., Pompeyo pudo formar una amplia clientela en toda Hispania. Aprovechando las clientelas hispanas que heredó de su padre, pudo mantener contacto directo con los pueblos de la Hispania Citerior en el transcurso del conflicto contra los sertorianos. Logró ganarse el apoyo de varios pueblos de la Celtiberia o de la Lusitania ${ }^{18}$, así como de los lacetanos o los indigetes, entre los que se encontraban los descendientes directos de los jinetes de la turma Salluitana a los que la gens Pompeia les era de sobra conocida ${ }^{19}$. Contaría para la defensa de su causa con el apoyo clientelar de varios pueblos hispanos entre los que figuraban los autrigones, los berones o los vascones $^{20}$.

Pompeyo procedió también a la puesta en práctica de los siguientes mecanismos: 1. Concesión del estatuto jurídico privilegiado. 2. Demarcación de nuevas fronteras. 3. Asentamiento de veteranos romano-itálicos que garantizasen la paz y el dominio romanos. 4. Fundación de nuevos núcleos urbanos o transformación de núcleos preexistentes. 5. Desarrollo de la red viaria. 6. Difusión y propaganda mediante la erección de trofeos ${ }^{21}$. Se puede pensar que la clientela pompeyana obscurecía el papel de otras existentes en Hispania, lo que no sería de extrañar, debido a que las fuentes que se han conservado presentan los hechos como si fuese un duelo personal entre pompeyanos y cesarianos, cuando en verdad se trató de una realidad más compleja.

Poco antes del enfrentamiento de llerda, el bando pompeyano logró ganarse la confianza de las tribus lusitanas, vetonas, celtíberas y cántabras. El bando pompeyano contó aproximadamente con cerca de diez mil legionarios de origen hispano al comienzo de la guerra civil. Los legionarios de origen hispano aparecen en las siguientes circunstancias ${ }^{22}: 1$. Como complemento de las legiones en el ejército de ocupación. 2. Miembros de la Legio Vernacula. 3. Legión reclutada por Q. Cassio Longino en 48 a.C. 4. Legión reclutada por los hijos de Pompeyo. 5. Dos cohortes coloniales que aparecen en Corduba. 6. Legiones heterogéneas reclutadas por los hijos de Pompeyo. 7. Los reclutamientos de Afranio y Petreyo entre lusitanos, celtíberos, cántabros y otros pueblos del norte, o de Varrón en la Hispania Ulterior. Sus nomina se registrarán de forma progresiva en los de la población indígena.

Los legados pompeyanos en Hispania, Afranio, Petreyo y Varrón, realizaron varios reclutamientos entre lusitanos, celtíberos, cántabros y otros pueblos del norte ${ }^{23}$, así como entre varios pueblos de regiones más meridionales ${ }^{24}$. Así, la presencia de auxiliares hispanos en el bando pompeyano fue decisiva en la resistencia contra

18 Las tribus de la Lusitania actuaron a favor de la causa del bando pompeyano en todo momento, quizás debido a las gestiones que años atrás llevó a cabo César en estos parajes durante la propretura.

19 Sall. Hist. 2.98.5.

20 Plut. Pomp. 18.1; Liv. Per. 91.22.10.

21 Sall. Hist. 3.89; Strab. 3.4.1, 3.4.7; Exuper. BC 56; Dio Cass. 41.24.3.

22 Roldán (1972: 109).

${ }_{23}$ Caes. Ciu. 1.38.3.

24 Caes. Ciu. 2.18.1; Bell.Hisp. 30.1. 
los efectivos cesarianos. Los hispanos más beneficiados por las gestiones llevadas a cabo por Pompeyo fueron, lógicamente, los pueblos de la Hispania Citerior. No obstante, las medidas de privilegio pompeyanas también alcanzaron la Hispania UIterior como lo prueba la concesión del estatuto privilegiado a los Balbo de Cádiz. Podemos apreciar, por tanto, cómo Pompeyo concedía el estatuto privilegiado de ciudadanía romana no tanto a colectividades sino a particulares y pequeños colectivos de las aristocracias y de las oligarquías locales, utilizando el derecho latino como instrumento de integración. Ahora bien, no todos los dependientes de Pompeyo en Hispania fueron indígenas, sino que sería comprensible que entre ellos figurase un numeroso colectivo de romanos e itálicos ${ }^{25}$.

Por otro lado, Pompeyo no sólo hizo de Numidia una variada fuente de ingresos, sino que la convirtió en una fuente inagotable de clientes donde poder estrechar pactos de amicitia. A diferencia de lo que había ocurrido en el Piceno o en la Galia Traspadana con sus predecesores, en el caso del reino de Numidia, había sido él mismo quien comenzó a construir una auténtica red clientelar fiel a su gens. Sus actuaciones se remontan a su participación en las campañas militares contra los marianistas en 81 a. C. Las relaciones fueron muy cordiales como demuestran las concesiones de ciudadanía romana a algunos habitantes de Utica ${ }^{26}$, siendo el responsable directo del reestablecimiento en el trono de Hiempsal II, depuesto anteriormente por el usurpador popular Hierbas, y de haber tomado las tierras de los gétulos, quienes las habían recibido de manos de Mario. Una de las consecuencias inmediatas de estas actuaciones fue que durante la guerra civil, Juba I ${ }^{27}$, hijo de Hiempsal II, prestase su apoyo incondicional al bando pompeyano ${ }^{28}$. Sin embargo, el transcurso del Bellum Civile provocó que las dos Numidias acabasen por ser totalmente filocesarianas ${ }^{29}$. Los monarcas mauritanos decidieron apoyar a César en 49 a.C. debido a que los monarcas númidas, sus más directos enemigos, estaban aliados con los Escipiones, partidarios del bando pompeyano. Así, las actuaciones de Boco II en el Bellum Alexandrinum invadiendo Numidia o la presencia decisiva de Bogud ${ }^{30}$ durante la batalla de Munda están más que aceptadas. Tales fidelidades políticas quedan recogidas por Dión Casio ${ }^{31}$ : «...en cuanto a Juba, recibió honores por parte de Pompeyo y los demás senadores que estaban en Macedonia y fue nombrado rey; pero fue llamado a juicio por César y los que estaban en Roma y se le declaró enemigo, mientras que Boco y Bogud fueron nombrados reyes porque eran enemigos de Juba».

25 La existencia del gentilicio Pompeius debe interpretarse más propiamente como un elemento de aculturación de la población indígena que como un elemento identificador de la clientela de la gens Pompeia.

26 Cic. Balb. 51.

27 Bengtson (1991: 289-97).

28 Caes. Ciu. 2.25.4.

29 Alföldi (1958: 103-108). Consideramos que no todos los pueblos númidas apoyaron al bando pompeyano durante la contienda, ya que un gran número de gétulos y varias tribus númidas abandonaron el bando pompeyano para apoyar al cesariano (Afr. 32.3; 56.3).

30 Gozalbes (1994: 287-93).

31 Dio Cass. 41.42.7. 
Los reyes y dinastas de Oriente se mantuvieron fieles a Pompeyo mientras éste se mantuvo en el poder. Por eso no los podemos considerar como auténticos clientes, porque de haberlo sido habrían defendido siempre la causa pompeyana. Los efectivos del monarca mauritano Bogud se aliaron finalmente con César siendo crucial su participación tanto en Hispania como en África.

\section{LOS HIJOS DE CN. POMPEYO MAGNO}

Muerto Cn. Pompeyo Magno, la resistencia pompeyana quedó en manos de Gneo Pompeyo y Sexto Pompeyo, quienes hicieron de Hispania el auténtico centro de operaciones contra las actuaciones cesarianas. Con una expedición formada por treinta navíos y tres mil hombres, entre los que figuraban esclavos, Gneo Pompeyo zarpó de Utica en dirección a Mauritania para después desembarcar en Hispania ${ }^{32}$. En los últimos meses de 47 a.C. saliendo de Ebusus, llegó y asedió la estratégica ciudad de Carthago Nova. Proclamado imperator, inició una serie de campañas triunfales por toda la Hispania Ulterior. Además, en poco tiempo logró reunir a todas las tropas propompeyanas supervivientes de la batalla de Thapsos. La reacción cesariana en Hispania no se dejó notar hasta 46 a.C., ya que primero era necesario acabar con las amenazas presentes en la costa africana, así como en Italia y Sicilia.

Siguiendo los testimonios de Dion Casio ${ }^{33}$, Gneo Pompeyo optó por apelar a la fides de las ciudades, concediendo todo tipo de favores para forjar una red clientelar y de amistad digna con la que ejercer la resistencia anticesariana. Con estos métodos no sólo forjó nuevas clientelas en la Hispania Ulterior, sino que también sumó a su causa las que habían prestado servicio en la Hispania Citerior ${ }^{34}$. Las actuaciones de su progenitor le permitieron poder contar con clientelas en la práctica totalidad de Hispania, razón clave por la que decidió convertirla en centro de operaciones.

Por otro lado, ¿cuál fue el control territorial con el que contó Sexto Pompeyo en Hispania? En primer lugar, podemos afirmar que el conjunto de fuentes grecolatinas no ofrece una información sobre cuál fue la reconquista que llevó a cabo. En este sentido, Apiano ${ }^{35}$ afirma, sin indicar el nombre de los núcleos urbanos, que tomó posesión de muchas ciudades. Por su parte, Dion Casio ${ }^{36}$ afirma que logró conquistar Carthago Nova, Barea y Carteia. En nuestra opinión sería capaz de sumar a su causa un número mayor de ciudades debido al filopompeyanismo de la práctica totalidad de Hispania. Tras la batalla de Munda, Sexto Pompeyo escapó rumbo a la Hispania Citerior llegando a la Lacetania, zona poco romanizada donde contaba con las clientelas forjadas por sus predecesores. Dominada ésta, logró dominar todo el valle del Ebro.

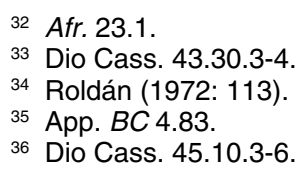


Si hubo un territorio que apoyó sin ninguna duda la causa de Sexto Pompeyo ese fue el de Cartagena, ciudad que tomó tras derrotar a Polión. La información aportada por la numismática nos hace plantearnos la teoría de que Sexto Pompeyo lograse el dominio sobre Urso, Myrtilis o Baelo. Todas ellas aparecen en las fuentes como partidarias de Sexto Pompeyo ${ }^{37}$. Resulta razonable que lograse dominar la Lusitania como parecen demostrar las cecas de Baesuri o Ketovion, relacionadas de algún modo con Sexto Pompeyo ${ }^{38}$. Igualmente, también dominaría el territorio de Nabrissa e Ilurco. Tomada Carthago Nova, emprendió su marcha hacia la Hispania Ulterior tomando otras muchas ciudades y soldados logrando dominar la costa levantina. El Bellum Hispaniense presenta como ciudades filopompeyanas a Urso, Carteia ${ }^{39}$, Hispalis, Italica ${ }^{40}$, Hasta, Ucubi, Ategua, Ventipo, Saguntum y Aspavia. Así, podemos afirmar que el hecho de que Sexto Pompeyo fuese expulsado de Hispania por la vía diplomática y no por las armas deja ver el potencial con el que contaba en Hispania.

\section{RELACIONES CESARIANAS DURANTE EL CONFLICTO}

Para poder comprender la posición que tomaron determinados individuos o colectivos durante el transcurso del conflicto, es necesario analizar la gestión políticoadministrativa y las relaciones sociales llevadas a cabo por César en Hispania desde 69 a.C., año en que ejerce la cuestura, hasta los idus de Marzo, analizando las redes clientelares creadas durante estos años, hecho que contribuyó en gran medida a ejercer una fuerte romanización de estas tierras mediante la concesión del estatuto jurídico privilegiado, conversión de antiguos núcleos indígenas en colonias o en municipios, o bien mediante el establecimiento de soldados veteranos y colonos de la plebe urbana de Roma en determinadas ciudades ${ }^{41}$.

En recompensa a los servicios militares o en recompensa del fervor concedió privilegios como la ciudadanía procediendo, paralelamente, a llevar a la práctica un programa socio-político de establecimiento de colonias y municipios para confirmar el cesarismo en Hispania, además de un gran número de adeptos capaces de luchar por su causa. La historiografía coincide al señalar que los intereses de César en Hispania eran múltiples, aunque estos pueden resumirse en consolidar su prestigio y su red clientelar, así como en conseguir un amplio territorio rico en recursos con una magnífica situación estratégica donde poder llevar a cabo sus operaciones militares y donde poder asentar a sus veteranos de guerra, pudiendo neutralizar a los efectivos militares de la Hispania Citerior, zona aún muy ligada a la causa pompeyana.

37 Caes. Bell. Hisp. 22.

38 Canal Junco (1994: 258).

39 Cic. Att. 15.20.3.

40 Caes. Ciu. 20.6; Bell.Alex. 57-58.

41 Henderson (1942: 5-13), Novillo (2009: 281-96). 
Como cuestor de la Hispania Ulterior en 69 a.C. y como gobernador en 61 a.C. de la misma provincia, consiguió crear una eficaz red clientelar utilizando fórmulas similares a las empleadas por la gens Pompeia. Siendo cuestor trató de aumentar las clientelas mediante la asistencia a los tribunales y por medio de la munificencia. Durante su estancia como cuestor mantuvo estrechas relaciones de amistad con las familias más influyentes de Cádiz y de Córdoba llevando a cabo el reclutamiento de nuevas tropas ${ }^{42}$. Estas primeras relaciones con la población cordobesa y gaditana se vieron consolidadas años más tarde durante el ejercicio de su propretura en 61 a.C. Los Balbo desempeñaron un papel crucial en beneficio de César siendo sus mecenas. Esta sería una de las razones fundamentales por las que César concedió el estatuto de ciudadanía romana a Gades.

El elemento indígena constituyó siempre un importante componente militar para el ejército romano. Así se aprecia en la propretura de César al añadir junto a sus legiones una tercera indígena ${ }^{43}$. En realidad, sólo ejerció un dominio real hasta las tierras regadas por el Tajo. Como procónsul se fijó como objetivo el pacificar y dominar los pueblos de la Lusitania, concretamente los ubicados en el entorno de la Sierra de la Estrella (Mons Herminius), dirigiéndose en primer lugar hacia $\mathrm{Me}$ tellinum, región en la que se refugiaban los lusitanos que escapaban de Roma. En estos abruptos parajes comenzó a trabar relaciones con la población lusitana, tratando de sumarlos a su causa y convertirlos de forma colectiva en sus clientes mediante la concesión de una serie de mejoras sociales y político-administrativas. Ateniéndonos a los restos materiales y a las estructuras arquitectónicas que han sobrevivido al paso de los años, entendemos que en estos precisos momentos se dibujaron por vez primera los patrones cesarianos del núcleo urbano fijo y estable, al ordenar a la población lusitana que abandonase las alturas del monte para trasladarse al terreno llano.

Finalizada con éxito la expedición contra los lusitanos, aprovechó las flotas enviadas por los gaditanos para emprender una nueva expedición hacia las ricas costas galaicas, llegando a Brigantium, cuyos habitantes se rindieron a los ejércitos cesarianos sin oponer ningún tipo de resistencia. Estas y otras actuaciones le permitieron ser aclamado por sus soldados como imperatory poder contar con la fidelidad de determinados núcleos como Corduba, Carmo, Hispalis o Gades durante el Bellum Civile. El hecho de liberar a las comunidades de la Hispania Ulterior de los tributos que Metelo había impuesto o de reestablecer la concordia entre las ciudades, fue lo que le permitió poder contar con el favor de varias ciudades. Aunque en primer término pueda parecer que las gestiones cesarianas únicamente surtieron efecto en tierras meridionales, en realidad no fue de tal modo porque impor-

42 Entre los gaditanos que mantuvieron estrechas relaciones y colaboraciones con César se encontraba L. Cornelio Balbo quien había obtenido, al igual que el resto de su familia, la ciudadanía romana por parte de Pompeyo gracias a su activa participación durante el conflicto sertoriano ingresando en el orden ecuestre. Los Balbo apoyaron en todo momento la causa cesariana, secundando los proyectos de César y prestando apoyo en todas las campañas bélicas.

43 Plut. Caes. 12. Sería César el primer romano en emprender una leva legionaria entre ciudadanos romanos no itálicos. 
tantes ciudades del valle del Ebro, como Osca, mostraron una enardecida fidelidad cesariana durante el conflicto. Consideramos que con César el derecho fue concedido de forma más o menos regular a los provinciales, siendo su obra entendida como una política de integración jurídica negociando con emigrantes itálicos e hispanos $^{44}$. Además, la base del programa cesariano era de tal firmeza que no se vio interrumpido tras su asesinato. En el último año de guerra César contaba con un ejército compuesto por ocho legiones y ocho mil jinetes como tropas auxiliares. Se trataba, además, de un ejército compuesto mayoritariamente de legionarios itálicos y auxiliares de caballería e infantería procedentes de la Galia, utilizando en menor medida el elemento militar hispano ${ }^{45}$.

La concesión del estatuto privilegiado permitió a César poder contar con un gran número de adeptos durante el transcurso del conflicto. Elevó al rango de municipio a núcleos que ya contaban con un importante número de personas dotadas del derecho ciudadano. Fueron muy numerosas las concesiones de privilegio jurídico a las poblaciones hispanas fieles o neutrales a su causa al elevar al rango de colonias de ciudadanos romanos a varios núcleos. A priori, un rasgo distintivo que podemos observar entre las colonias y los municipios cesarianos es que, mientras en las colonias imperaban los cánones militares sobre los civiles, en el caso de los municipios ocurría lo contrario. Hemos de tener presente que fueron varias las manumisiones que se llevaron a cabo condicionando el incremento de libertos. Consideramos que, en cierta medida, parte de los éxitos de César durante y tras el conflicto se deben a su política de atracción y de promoción jurídico-administrativa. En suma, la ventaja principal de César sobre Pompeyo no consistió sino en conseguir una amplia base de prestigio ante los hispanos y en emplear una sofisticada estrategia militar.

\section{LA CLEMENTIA CAESARIS}

La clementia era el término utilizado para designar una conducta o un comportamiento compasivo, benigno o misericordioso para con los demás. Se practicó durante todo el periodo tardorrepublicano, y más concretamente desde el final de las guerras civiles, sirviendo para designar la actitud que determinados generales mantuvieron con respecto a los derrotados ${ }^{46}$. La clemencia de César era conocida en todo el ejército pompeyano ${ }^{47}$, siendo muchos los que habiendo sido derrotados pidieron clemencia al dictador a cambio de servirle fielmente ${ }^{48}$. Fue una práctica muy utilizada por César, razón por la que se levantó el templo a la diosa Clementia. Aplicando ésta pudo hacerse con la voluntad de los derrotados y sumar así

\footnotetext{
44 Abascal y Espinosa (1989: 59).

45 Creemos que en su ejército no se incluyeron de forma generalizada a esclavos o libertos por no querer convertirlos en ciudadanos.

46 Hellegouarc'h (1972: 262).

47 Treu (1948: 197-217), Voi (1972: 121-25), Dahlmann (1980: 32-47), Caballero del Sastre (1998: 97-110).

48 Caes. Ciu. 1.72, 1.74.2, 1.85, 1.86, 2.28; Bell.Hisp. 19.4.
} 
nuevos efectivos como recogen los siguientes pasajes: «A partir de estos sucesos, aterrados aún más por la severidad de Petreyo, se mostraron proclives a la clemencia de C. Julio César» (App. BC. 2.143). «Nosotros, que hemos resistido el valor de tus legiones, esperado día y noche en los reparos el corte de las espadas y el tiro de los dardos, vencidos y desamparados de Pompeyo, rendidos a tu valor, pedimos la vida a tu clemencia y te suplicamos te muestres en la rendición de tus ciudadanos cual te has mostrado a los extranjeros». César le respondió: "Cual me he mostrado a los extranjeros, me mostraré en la rendición de los ciudadanos» (Bell.Hisp. 17).

\section{DESERCIONES}

Aunque se dieron en ambos bandos como una práctica muy común del ejército derrotado, las deserciones se produjeron mayoritariamente en el bando pompeyano con motivo de los avances y la presión militar cesariana ${ }^{49}$. Logrado el asedio de Massalia, varias ciudades de la Hispania Citerior abandonaron los apoyos a Afranio buscando con ello la alianza con César a cambio de asegurar sus líneas de aprovisionamiento ${ }^{50}$. Los miembros de las oligarquías locales y los particulares indígenas fueron los principales desertores. Estos servirían en numerosas ocasiones para informar a un bando $u$ otro de los movimientos y operaciones del bando contrincante, fenómeno que se aprecia en los siguientes pasajes: «Terminado el puente, ganadas en amistad cinco grandes ciudades, escueto el problema de abastecimiento, extinguidos los rumores de los refuerzos de legiones, que se decía se acercaban con Pompeyo por Mauritania, muchas ciudades más lejanas desertan de Afranio y buscan la amistad de César» (Caes. Ciu. 1.60). "Pues hasta ese momento nadie, o de infantería o de caballería, se había pasado de las filas de César a las de Pompeyo; aunque casi diariamente se pasaban de las de Pompeyo a las de César» (Caes. Ciu. 3.61).

\section{BIBLIOGRAFÍA}

ABASCAL, J. M. y ESPINOSA, U. (1989), La Ciudad Hispano-Romana. Privilegio y Poder, Logroño.

ALFÖLDI, A. (1958), «Juba I und die Pömpeianer in Afrika», Schwezer Münzlätter 8, 103-108. AMELA, L. (2000), «La Turma Salluitana y su relación con la clientela pompeyana», Veleia $17,79-92$.

BADIAN, E. (1958), Foreing Clientelae 264-70 B.C., Oxford.

BENGTSON, H. (1991), «L'aide militaire de Juba ler aux pompéiens pendant la guerre civile en Afrique du Nord (50-46 avant J.C.)", en Histoire et Archeologie de l'Afrique du Nord, Paris, 289-297.

BRUNT, P. A. (1965), «Italian aims at the time of the Social War», JRS 55, 90-109.

49 Caes. Ciu. 3.60.5; Bell.Hisp. 2.18.3, 2.19.3, 2.34.1-2

50 Caes. Ciu. 1.60 .5 
- (1969), «Amicitia in the Late Roman Republic», en Seager, R. ed., The Crisis of the Roman Republic, Cambridge, 199-218.

CABALLERO DEL SASTRE, E. et al. (1998), «La clementia Caesaris y el Pro Marcelo», Flllib 9, 97-110.

CANAL, P. (1994), Sexto Pompeyo en Hispania, Madrid.

COULANGES, F. (1890), Histoire des Institutions Politiques de l'Ancienne France. Vol. V. Les Origines du Système Féodal, Paris.

DAHLMANN, H. (1980), «Clementia Caesaris», en Rasmussen D. ed., Caesar, Darmstadt, 32-47.

GOZALBES, C. (1994), "La intervención de la Mauritania de Bogud en las Guerras Civiles romanas en la PHU», en Actas del II Congreso de Historia de Andalucía. Historia Antigua, Córdoba, 287-293.

HELLEGOUARC'H, H. (1972), Le Vocabulaire Latin des Relations et des Partis Politiques sous la République, Paris.

HENDERSON, M. I. (1942), «lulius Caesar and Latium in Spain», JRS 32, 1-13.

NICOLS, J. (1980), «Pliny and the patronage of communities», Hermes 108.3, 365-85.

NOVILLO, M. A. (2009): «La administración cesariana en la Provincia Hispania Ulterior. la integración de la población hispana en el modelo de la civitas romana», en Bravo, G. y González Salinero, R. eds., Formas de Integración en el Mundo Romano, Madrid, 28196.

RAWSON, E. (1978), "Caesar, Etruria and the disciplina Etrusca», JRS 68, $132-52$.

ROLDÁN HERVÁS, J. M. (1972), «El elemento indígena en las guerras civiles en Hispania: aspectos sociales», HA 2, 77-123.

SERRANO, J. M. (1989), «Documentos adicionales relativos a la amicitia», Habis 20, 175-84. TREU, M. (1948), «Zur clementia caesaris», Maia 5, 197-217.

VOI, G. (1972), "Clementia e lenitas nella terminologia e nella propaganda cesariana», en Contrib. Ist. Storia Ant. I, 121-25. 